\title{
Predicting incident fatty liver using simple cardio-metabolic risk factors at baseline
}

\author{
Ki-Chul Sung ${ }^{1,8^{*}}$, Bum-Soo Kim', Yong-Kyun Cho², Dong-il Park², Sookyoung Woo ${ }^{3}$, Seonwoo Kim³ \\ Sarah $\mathrm{H}$ Wild ${ }^{4}$ and Christopher D Byrne $e^{5,6,7^{*}}$
}

\begin{abstract}
Background: Non alcoholic fatty liver disease (NAFLD) is associated with increased risk of type 2 diabetes and chronic liver disease but identifying patients who have NAFLD without resorting to expensive imaging tests is challenging. In order to help identify people for imaging investigation of the liver who are at high risk of NAFLD, our aim was to: a) identify easily measured risk factors at baseline that were independently associated with incident fatty liver at follow up, and then b) to test the diagnostic performance of thresholds of these factors at baseline, to predict or to exclude incident fatty liver at follow up.
\end{abstract}

Methods: 2589 people with absence of fatty liver on ultrasound examination at baseline were re-examined after a mean of 4.4 years in a Korean occupational cohort study. Multi-variable logistic regression analyses were used to identify baseline factors that were independently associated with incident fatty liver at follow up. The diagnostic performance of thresholds of these baseline factors to identify people with incident fatty liver at follow-up was assessed using receiver operating characteristic (ROC) curves.

Results: 430 incident cases of fatty liver were identified. Several factors were independently associated with incident fatty liver: increased triglyceride (per mmol// increase) OR 1.378 [95\%Cls 1.179, 1.611], p < 0.0001; glucose (per mmol/l increase) OR 1.215 [95\%Cls 1.042, 1.416], $p=0.013$; waist (per $\mathrm{cm}$ increase) OR 1.078 [95\%Cls 1.057, 1.099], $p<0.001$; ALT (per IU/L increase) OR 1.009 [95\%Cls 1.002, 1.017], $p=0.016$; and platelets (per $1 \times 10^{9} / \mathrm{L}$ increase) OR $1.004[1.001,1.006], p=0.001$; were each independently associated with incident fatty liver. Binary thresholds of the five factors were applied and the area under the ROC curve for incident fatty liver was 0.75 (95\% Cl 0.72-0.78) for the combination of all five factors above these thresholds.

Conclusion: Simple risk factors that overlap considerably with risk factors for type 2 diabetes allow identification of people at high risk of incident fatty liver at who use of hepatic imaging could be targeted.

Keywords: Non alcoholic fatty liver disease, Fatty liver, Etiology, Risk prediction, Metabolic syndrome

\section{Background}

Prevalence estimates for non alcoholic fatty liver disease (NAFLD) range from $17 \%$ to $33 \%$ in general populations in Western countries [1,2] and it is predicted that NAFLD will soon become the most important contribution to demand for liver transplantation [3]. NAFLD is

\footnotetext{
* Correspondence: kcmd.sung@samsung.com; c.d.byrne@soton.ac.uk 'Division of Cardiology, Department of Medicine, Kangbuk Samsung Hospital, Sungkyunkwan University School of Medicine, Seoul, Republic of Korea

${ }^{5}$ Nutrition and Metabolism Unit, Faculty of Medicine, University of Southampton, IDS Building, Southampton General Hospital, MP 887 Southampton, UK

Full list of author information is available at the end of the article
}

also associated with type 2 diabetes, cardiovascular disease $[4,5]$ and insulin resistance [6] in muscle, adipose and liver [7].

Patients with possible NAFLD are treated by a range of specialists [8] who have to decide which patients are at sufficiently high risk to investigate further to make the diagnosis. Readily available single anthropometric tests or blood tests lack sufficient sensitivity or specificity to identify patients with NAFLD. For example, a body mass index (BMI) $\geq 25 \mathrm{~kg} / \mathrm{m}^{2}$ as a measure of overweight or obesity, is not specific for NAFLD, because not all overweight or obese people have fatty liver; and an alanine transaminase (ALT) value $>31 \mathrm{U} / 1$ in men and

\section{Biomed Central}


$>19 \mathrm{IU} / \mathrm{l}$ in women is fairly sensitive but is not a specific test for NAFLD [9]. It is therefore difficult to select appropriate patients for expensive radiological diagnostic imaging tests such as ultrasound, because single tests lack sufficient sensitivity and specificity to identify high risk patients. Although other more sensitive techniques than ultrasound are available for detecting liver fat, e.g. proton magnetic resonance spectroscopy (MRS) [10,11], MRS is an expensive diagnostic test and is also imperfect at identifying the extent or severity of NAFLD. Consequently, ultrasound is usually the preferred initial radiological test employed by most clinicians to establish the presence of moderate or large amounts of liver fat.

There is increasing interest in developing algorithms or risk scores for identifying liver fat, because accumulation of liver fat or some component of fat metabolism is the prerequisite for progression of NAFLD to steatohepatitis and fibrosis [12]. Several investigators have undertaken cross sectional studies examining relationships between risk factors and prevalent fatty liver [13-17], but the ability of these risk factors, or algorithms, to predict incident fatty liver in a prospective study is uncertain.

Recently, in a cross sectional study of people with and without diabetes, and people recruited from gastroenterology clinics, Kotronen et al. developed a NAFLD liver fat score based on serum insulin, aspartate transaminase (AST), ALT concentrations, presence or absence of metabolic syndrome and type 2 diabetes. These investigators validated the fat score against the presence and quantity of hepatic fat as assessed by MRS [18]. In this study, the optimum liver fat score cut-point had $86 \%$ sensitivity and 71\% specific for predicting NAFLD [18]. Although these data suggest an excellent performance of the liver fat score, an accompanying editorial suggested the score was 'not ready for prime time'[19], not least because of the cross sectional study design and the limited potential applicability of these findings to general populations as some participants were recruited from hospital clinics.

We have undertaken a cohort study of people who were shown to be free from hepatic steatosis on ultrasound examination at baseline, and who had a further ultrasound examination at follow up an average of 4.4 years later. We used these data to identify individuals who had developed new (incident) fatty liver during the follow up period. Since there are no guidelines to help identify which patients should undergo ultrasound testing to diagnose fatty liver, the aim of our study was to: a) identify easily measured risk factors at baseline that were independently associated with incident fatty liver at follow up, and then b) to test the diagnostic performance of thresholds of these factors to predict or to exclude incident fatty liver at follow up.

\section{Methods}

Electronic medical records were used to identify 4463 participants from a cohort of Korean employees who had had an occupational health check in 2003-2005 that included collection of data on anthropometry and liver ultrasound. Information on smoking history (never, current, or past) and whether individuals participated in any regular exercise was obtained by questionnaire. A follow up examination was performed in 2008 and 2009 at Kangbuk Samsung Hospital in Seoul, Korea and people without fatty liver at baseline were only included if they had a further ultrasound at follow-up (Figure 1). The institutional review board of Kangbuk Samsung Hospital approved this study.

We excluded participants with positive serologic marker for hepatitis B $(n=188)$ or hepatitis $C$ virus $(n=13)$ and alcohol consumption in excess of $20 \mathrm{~g} /$ day $(\mathrm{n}=531)$. Regular exercise and smoking status were assessed by a physician administered questionnaire. Individuals were asked if they had participated in any regular exercise at

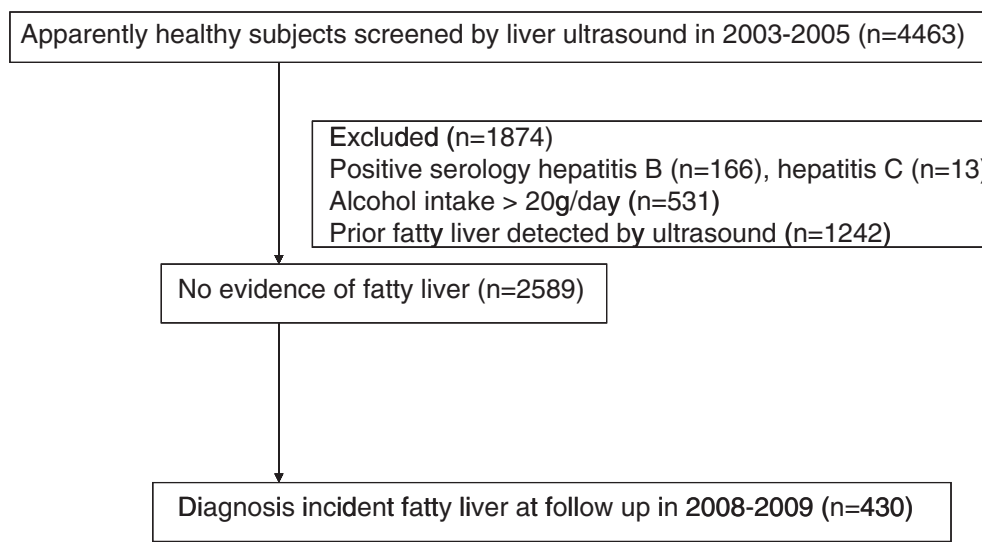

Figure 1 Flow chart summarizing the selection of study participants. 
least once a week and smoking status was classified as never, ex and current smoker. Data were specifically requested from the central source on those individuals who had a complete data-set for all these measurements.

Plasma and serum were collected after at least $12 \mathrm{~h}$ of fasting. Glucose was measured using the hexokinase method (Advia 1650 Autoanalyzer; Bayer Diagnostics, Leverkusen, Germany). Insulin was measured with an immunoradiometric assay (Biosource, Nivelle, Belgium) (intra- and interassay coefficient of variation of 2.1-4.5\% and $4.7-12.2 \%$, respectively). An enzymatic calorimetric test was used to measure total cholesterol and triglyceride concentrations. High density lipoprotein cholesterol (HDLc) concentration was measured by the selective inhibition method and a homogeneous enzymatic calorimetric test was used to measure low density lipoprotein cholesterol (LDLc) concentration (Advia 1650 Autoanalyzer). High-sensitivity C-reactive protein (hs-CRP) was analysed by performing particle-enhanced immunonephelomety using the BN System (Dade Behring, Marburg, Germany). HOMA-IR (Homeostasis Model Assessment of Insulin Resistance) was calculated as a measure of insulin resistance [20]. Abdominal ultrasonography (Logic Q700 MR; GE, Milwaukee,WI, USA) was performed in all subjects by experienced clinical radiologists and fatty liver was diagnosed based on known standard criteria, including hepatorenal echo contrast, liver brightness, and vascular blurring, using a 3.5 $\mathrm{MHz}$ probe [21].

\section{Risk prediction modeling for fatty liver comparing established and population-specific newly optimized thresholds}

All the observations including ultrasound and blood tests that are included in the analyses were undertaken at base-line and data from a further ultrasound performed at follow up was available for people without fatty liver at baseline. Established thresholds for metabolic risk factors (waist, glucose and triglyceride) were identified according to the Joint Interim Statement 2009 criteria for the metabolic syndrome (MetS) [22].

For ALT, we used sex specific cut-offs for normal liver function that have been shown to exclude NAFLD [9]. For platelet number, we used the lower limit of normal for Kangbuk Samsung Hospital, Korea as the cut point $\left(158 \times 10^{9} / \mathrm{l}\right)$.

Additionally, we also identified new populationspecific optimized thresholds of each variable that showed the highest accuracy (based on the area under receiver operating curves, AUROC) for identifying people at high risk of incident fatty liver, and the best accuracy (NPV\%) for excluding incident fatty liver at follow up; using the Youden index method [23].

\section{Validation of the performance of established thresholds and population-specific thresholds in training and validation data-sets}

We established training and validation data-sets to validate the performance of the established and new population-specific thresholds. To develop training and validation data-sets, we split the data for each stratum into training and validation data-sets, using random sampling methodology to ensure similar distribution of sex and body mass index (BMI) between both data subsets.

\section{Statistical analysis}

The descriptive statistics for continuous variables are presented using means and standard deviations (SDs) for normally distributed variables and medians and ranges for non-normally distributed variables. Categorical variables were described using frequencies (percentages). Continuous variables were compared between two groups using $t$-test or Mann-Whitney $U$ tests and categorical variables were analyzed using chi-squared test or Fisher's exact test. To identify baseline factors that were independently associated with incident fatty liver at follow up, logistic regression analysis was undertaken, and the odds ratio (OR) and 95\% confidence intervals for incident fatty liver were calculated for continuous variables. The diagnostic performance of binary criteria for baseline biochemical and anthropometric tests that were independently associated with incident fatty liver at follow up was assessed using ROC curves using both established cut-points used to define the MetS and cutpoints identified within the study population. All statistical analyses were performed using SPSS 17.0 (SPSS Inc., Chicago, IL, USA) and Stata 11.0 (StataCorp LP, 4905 Lakeway Drive College Station, Texas 77845 USA).

\section{Results}

We identified 988 people with prevalent fatty liver and 2589 people who did not have ultrasound evidence of fatty liver, positive hepatitis B or C serology or excessive alcohol consumption at baseline examination. Of these 2589 people, 430 developed incident fatty liver, during the mean follow-up period of average 4.37 years (1596 days). Table 1 shows a comparison of baseline characteristics (a) between individuals with and without fatty liver at baseline and (b) between individuals with and without incident fatty liver at follow up. Mean HOMA-IR was higher in the group with fatty liver compared with the group without fatty liver at baseline $(2.69 \pm 1.16$ v. $1.95 \pm 0.69, \mathrm{p} \leq 0.0001)$. There were also a higher percentage of people with MetS in the group with fatty liver compared with the group without fatty liver at baseline $(29.7 \%$ v. $5.2 \%, \mathrm{p} \leq 0.0001)$. A higher proportion of men (23.4\%) than women (9.7\%) developed incident 
Table 1 Comparison of baseline characteristics (a) between individuals with and without fatty liver at baseline and (b) between individuals with and without incident fatty liver at follow up

\begin{tabular}{|c|c|c|c|c|c|c|}
\hline & \multicolumn{3}{|c|}{ Baseline fatty liver status (a) } & \multicolumn{3}{|c|}{ Follow up fatty liver status (b) } \\
\hline & Absent & Present & (a) & Absent & Present & (b) \\
\hline & & & $\overline{p \text { value }}$ & & & p value \\
\hline$n$ & 2589 & 988 & & 2159 & 430 & \\
\hline Age (y) & $46.27 \pm 8.51$ & $48.08 \pm 8.69$ & $<0.0001$ & $46.06 \pm 8.57$ & $47.32 \pm 8.11$ & 0.005 \\
\hline$\%$ Men & $1305(50.4 \%)$ & $800(81.0 \%)$ & $<0.0001$ & $999(46.3 \%)$ & $306(71.2 \%)$ & $<0.0001$ \\
\hline BMI $\left(\mathrm{kg} / \mathrm{m}^{2}\right)$ & $22.94 \pm 2.58$ & $26.10 \pm 2.42$ & $<0.0001$ & $22.66 \pm 2.53$ & $24.33 \pm 2.32$ & $<0.0001$ \\
\hline Waist & $77.00 \pm 8.44$ & $87.52 \pm 6.68$ & $<0.0001$ & $75.93 \pm 8.25$ & $82.36 \pm 7.22$ & $<0.0001$ \\
\hline \multicolumn{7}{|l|}{ Exercise } \\
\hline None & $817(31.5 \%)$ & $270(27.3 \%)$ & .002 & $689(31.9 \%)$ & $128(29.8 \%)$ & 0.81 \\
\hline$\leq 1$ time/week & $359(13.9 \%)$ & 183(18.5\%) & & $297(13.8 \%)$ & $62(14.4 \%)$ & \\
\hline$>1$ time/week & $1413(54.6 \%)$ & $535(54.1 \%)$ & & $1173(54.3 \%)$ & $240(55.8 \%)$ & \\
\hline \multicolumn{7}{|l|}{ \% Smoker } \\
\hline Never & $1821(70.3 \%)$ & 490(49.6\%) & $<0.0001$ & $1581(73.2 \%)$ & $240(55.8 \%)$ & $<0.0001$ \\
\hline Past & $293(11.3 \%)$ & $238(24.1 \%)$ & & $215(10.0 \%)$ & $78(18.1 \%)$ & \\
\hline Current & $475(18.3 \%)$ & $260(26.3 \%)$ & & $363(16.8 \%)$ & $112(26.0 \%)$ & \\
\hline Alcohol (gm/day) & $5.81 \pm 7.07$ & $7.68 \pm 7.53$ & $<0.0001$ & $5.37 \pm 6.82$ & $8.01 \pm 7.84$ & $<0.0001$ \\
\hline SBP $(\mathrm{mmHg})$ & $115.23 \pm 13.82$ & $112.39 \pm 15.00$ & $<0.0001$ & $114.60 \pm 13.49$ & $118.36 \pm 14.97$ & $<0.0001$ \\
\hline $\mathrm{DBP}(\mathrm{mmHg})$ & $74.64 \pm 9.89$ & $80.08 \pm 10.08$ & $<0.0001$ & $74.06 \pm 9.71$ & $77.53 \pm 10.25$ & $<0.0001$ \\
\hline Triglyceride(mmol/L)Median[IQR] & $1.10[0.80,1.51]$ & $1.80[1.33,2.44]$ & $<0.0001$ & $1.04[0.77,1.41]$ & $1.38[1.04,1.91]$ & $<0.0001$ \\
\hline $\mathrm{HDL}-\mathrm{C}(\mathrm{mmol} / \mathrm{L})$ & $1.54 \pm 0.30$ & $1.40 \pm 0.23$ & $<0.0001$ & $1.56 \pm 0.31$ & $1.47 \pm 0.27$ & $<0.0001$ \\
\hline $\mathrm{HsCRP}(\mathrm{mg} / \mathrm{L})$ & $0.82 \pm 1.43$ & $1.38 \pm 1.75$ & $<0.0001$ & $0.77 \pm 1.40$ & $1.03 \pm 1.58$ & $<0.0001$ \\
\hline Platelets $\left(10^{9} / \mathrm{L}\right)$ & $246.14 \pm 53.10$ & $235.32 \pm 50.57$ & $<0.0001$ & $244.73 \pm 52.63$ & $253.16 \pm 54.90$ & 0.003 \\
\hline Albumin (g/L) & $45.6 \pm 2.4$ & $46.7 \pm 2.5$ & $<0.0001$ & $45.4 \pm 3.0$ & $45.9 \pm .2 .3$ & $<0.0001$ \\
\hline ALT (U/L) & $21.44 \pm 15.19$ & $38.39 \pm 22.02$ & $<0.0001$ & $20.38 \pm 11.67$ & $26.69 \pm 25.95$ & $<0.0001$ \\
\hline AST (U/L) & $22.14 \pm 7.11$ & $27.99 \pm 10.87$ & $<0.0001$ & $21.87 \pm 6.93$ & $23.46 \pm 7.83$ & $<0.0001$ \\
\hline AST/ALT ratio & $1.17 \pm 0.36$ & $0.82 \pm 0.27$ & $<0.0001$ & $1.19 \pm 0.36$ & $1.03 \pm 0.33$ & $<0.0001$ \\
\hline AST/platelet index & $9.46 \pm 3.97$ & $11.62 \pm 5.81$ & $<0.0001$ & $9.38 \pm 3.74$ & $9.86 \pm 4.95$ & 0.06 \\
\hline Fasting Glucose(mmol/L) & $5.10 \pm 0.66$ & $5.55 \pm 1.13$ & $<0.0001$ & $5.06 \pm 0.56$ & $5.26 \pm 1.01$ & $<0.0001$ \\
\hline Fasting Insulin (pmol/L) & $59.48 \pm 18.65$ & $75.39 \pm 27.89$ & $<0.0001$ & $58.91 \pm 18.18$ & $62.28 \pm 20.64$ & 0.002 \\
\hline HOMA-IR & $1.95 \pm 0.69$ & $2.69 \pm 1.16$ & $<0.0001$ & $1.92 \pm 0.66$ & $2.10 \pm 0.81$ & $<0.0001$ \\
\hline MetS, n (\%) & $135(5.2 \%)$ & $293(29.7 \%)$ & $<0.0001$ & $92(4.3 \%)$ & $43(10.0 \%)$ & $<0.0001$ \\
\hline
\end{tabular}

Data are presented as mean (SD), except where indicated.

fatty liver $(\mathrm{p}<0.0001)$. At baseline, there was a higher HOMA-IR, and a higher prevalence of MetS, in the people that developed fatty liver during the follow up period $(n=430)$ compared with those people who remained free from developing fatty liver $(n=2159)$ (Table 1).

As several individual cardio-metabolic risk factors are common to insulin resistance, MetS and type 2 diabetes, we investigated the relationship between individual cardio-metabolic risk factors at baseline and incident fatty liver at follow up. The results of analyses in which all potential baseline cardio-metabolic risk factors were entered into a logistic regression model with incident fatty liver as the outcome are shown in Table 2. Age, sex, hsCRP, blood pressure, HDLc, and smoking did not have statistically significant independent associations with incident fatty liver in the fully adjusted model. In contrast, glucose, waist circumference, triglyceride concentration, alanine transaminase (ALT) and platelet number were all independently associated with incident fatty liver in this model.

The significant variables identified from regression analysis (shown in Table 2) were selected for ROC curve analyses. The performance of these variables in risk prediction modeling for incident fatty liver is summarized in Table 3. The sensitivity of established thresholds 
Table 2 Odds ratios derived from multivariable logistic regression for the association between age, sex and cardio-metabolic risk factors at baseline and incident fatty liver at follow-up

\begin{tabular}{lcc}
\hline & \multicolumn{2}{c}{ Incident fatty at follow up } \\
\cline { 2 - 3 } & OR $[95 \%$ Cls $]$ & p value \\
\hline Age (per year) & $0.990[0.977,1.004]$ & 0.176 \\
Male sex & $1.103[0.795,1.531]$ & 0.557 \\
Glucose (per mmol/l increase) & $1.215[1.042,1.416]$ & 0.013 \\
Insulin (per pmol/L increase) & $1.002[0.996,1.008]$ & 0.436 \\
hsCRP (per mg/l increase) & $1.286[0.632,2.615]$ & 0.488 \\
Triglyceride (per mmol// increase) & $1.378[1.179,1.611]$ & $<0.0001$ \\
HDLc (per mmol/l increase) & $0.821[0.545,1.236]$ & 0.345 \\
ALT (per IU/L increase) & $1.009[1.002,1.017]$ & 0.016 \\
Platelets (per 1x10 $/ L$ increase) & $1.004[1.001,1.006]$ & 0.001 \\
Waist (per cm increase) & $1.078[1.057,1.099]$ & $<0.0001$ \\
Smoking (current vs past/never & $0.923[0.696,1.225]$ & 0.579 \\
DBP (per mmHg increase) & $1.003[0.990,1.015]$ & 0.656 \\
\hline
\end{tabular}

*or SBP OR [95\%Cls] $=1.002[0.993,1.011], p=0.65$.

Model adjusted for all of above variables.

(defined in methods) of the individual risk factors to identify a group at high risk of incident fatty liver are shown in Table 3 . These thresholds were very good at identifying people at low risk of incident fatty liver (negative predictive values (NPVs) for all variables were between $78.9 \%$ and $92.2 \%$ ).

Since there has been recent debate as to whether there should be population-specific thresholds for waist circumference as a key feature of the metabolic syndrome, we tested the diagnostic performance of populationspecific thresholds that showed the best discrimination for each of the five factors (waist circumference, glucose, triglyceride, ALT and platelets), to identify or exclude incident fatty liver. The results of applying the newly defined population-specific thresholds of each of these variables are also shown in Table 3. These data demonstrated that, for this population, lower thresholds of glucose $\left(5.0 \mathrm{mmol} . \mathrm{l}^{-1}\right)$, waist circumference $(78.8 \mathrm{cms}$ (men) and $73.6 \mathrm{cms}$ (women)), triglyceride $\left(1.1 \mathrm{mmol}^{-1} \mathrm{l}^{-1}\right.$, ALT (24 IU. $\mathrm{l}^{-1}$ (men), and $13 \mathrm{IU} . \mathrm{l}^{-1}$ (women)) and a higher platelet count $\left(262 \times 10^{-9} .1^{-1}\right)$ than the established or laboratory based thresholds), resulted in slightly better diagnostic performance of each test to identify a high risk group for fatty liver. These population-specific thresholds also improved performance of each test to exclude fatty liver (with the exception of ALT in men).

We examined the diagnostic performance of combinations of the variables (waist circumference, and concentrations of glucose, triglyceride and ALT, and platelet count) to identify high risk of incident fatty liver, using both the established, and the population-specific measurement thresholds. When all five risk factors (waist circumference, and concentrations of glucose, triglyceride and ALT, and platelet count) were combined, for established thresholds, the area under receiver operator curve (AUROC) value for incident fatty liver was $0.65(95 \%$ CI $0.61,0.69)$, with a positive predictive value (PPV) of 50.0\% (95\%CI 15.7, 84.3) and an NPV of $84.0 \%$ (95\%CI 81.9, 86.0); whereas for the population-specific thresholds, the AUROC for incident fatty liver was $0.75(95 \% \mathrm{CI} 0.72,0.78)$, with a PPV of $40.0 \%$ (95\%CI 15.7, 84.3) and an NPV of $85.4 \%$ (95\%CI 83.3, 87.3). We used the population-specific criteria to calculate the number and proportion of people who did not have fatty liver at baseline who met all five thresholds or who met the ALT threshold alone as these criteria are most likely to be applied in clinical practice to inform a decision to request a liver ultrasound examination. The number (\%) of people who met all five specific criteria was $87(3.4 \%)$ whereas 585 people (23\%) met the ALT threshold alone. Consequently, 585 people might be selected for an ultrasound examination, based upon the presence of an ALT concentration above the populationspecific threshold for ALT, whereas in contrast only 87 people would be selected for ultrasound investigation, by using the presence of all five criteria as the essential prerequisite.

Next we compared the diagnostic performance of combining the five factors in the training and the validation datasets. The performance of both sets of thresholds was very similar in the training and validation datasets (Table 4).

\section{Discussion}

Our study is the first to combine identification of risk factors with the subsequent testing of these factors in risk prediction modeling for incident fatty liver at follow up. Whilst others have examined relationships between risk scores (or cardio-metabolic risk factors) and prevalent fatty liver in cross sectional studies [13-17], we have examined the AUROC, NPV and PPV of risk factors identified at baseline, to predict incident cases of fatty liver at average follow up of 4.4 years. We show that combination of thresholds of five easily measured risk factors (waist circumference, glucose, ALT, platelets and triglyceride) allows identification of the small proportion of people at high risk of incident fatty liver who could then be selected for hepatic imaging tests. Such an approach would focus the use of relatively expensive non-invasive diagnostic imaging tests, such as ultrasound, and would be likely to increase the specificity of this imaging test to diagnose fatty liver, and lead to more cost effective use of limited resources. Additionally, our data suggest that in this cohort the population-specific thresholds are possibly better at diagnosing, or identifying, people at high risk of fatty liver (than the established thresholds). In contrast, 
Table 3 Comparison of the performance of key variables at baseline to identify incident fatty liver at follow up, using established and newly optimized thresholds of each variable*

\begin{tabular}{|c|c|c|c|c|c|c|}
\hline \multicolumn{7}{|c|}{ Established thresholds } \\
\hline Test & Cut point & $\begin{array}{l}\text { AUROC } \\
(95 \% \mathrm{CI})\end{array}$ & Sensitivity (\%) $(95 \% \mathrm{Cl})$ & $\begin{array}{c}\text { Specificity (\%) } \\
(95 \% \mathrm{Cl})\end{array}$ & PPV (\%) $(95 \% \mathrm{Cl})$ & NPV (\%) $(95 \% \mathrm{Cl})$ \\
\hline \multirow[t]{2}{*}{ Glucose } & $5.6(\mathrm{mmol} / \mathrm{l})$ & 0.56 & $24.9 \%$ & $86.6 \%$ & $26.4 \%$ & $85.7 \%$ \\
\hline & & $(0.53-0.59)$ & $(19.2 \%-31.3 \%)$ & $(84.5 \%-88.6 \%)$ & $(20.4 \%-33.1 \%)$ & $(83.5 \%-87.7 \%)$ \\
\hline \multirow[t]{4}{*}{ Waist } & 90 (cms) men & & $18.4 \%$ & $88.5 \%$ & $31.8 \%$ & $78.9 \%$ \\
\hline & & 0.56 & $(12.5 \%-25.6 \%)$ & $(85.4 \%-91.2 \%)$ & $(22.1 \%-42.8 \%)$ & $(75.3 \%-82.2 \%)$ \\
\hline & 80 (cms) women & $(0.53-0.58)$ & $29 \%$ & $90.5 \%$ & $24.7 \%$ & $92.2 \%$ \\
\hline & & & $(18.2 \%-41.9 \%)$ & $(87.8 \%-92.8 \%)$ & $(15.3 \%-36.1 \%)$ & $(89.7 \%-94.3 \%)$ \\
\hline \multirow[t]{2}{*}{ Triglyceride } & $1.7(\mathrm{mmol} / \mathrm{l})$ & 0.60 & $35.9 \%$ & $85.1 \%$ & $31.6 \%$ & $87.3 \%$ \\
\hline & & $(0.57-0.64)$ & $(29.4 \%-42.8 \%)$ & $(82.8 \%-87.1 \%)$ & $(25.8 \%-38.0 \%)$ & $(85.2 \%-89.3 \%)$ \\
\hline \multirow[t]{4}{*}{ ALT } & $30(\mathrm{IU} / \mathrm{I})$ & & $32.7 \%$ & $75.7 \%$ & $28.1 \%$ & $79.5 \%$ \\
\hline & (men) & 0.52 & $(25.2 \%-40.9 \%)$ & $(71.7 \%-79.4 \%)$ & $(21.5 \%-35.4 \%)$ & $(75.6 \%-83 \%)$ \\
\hline & $19(\mathrm{IU} / \mathrm{l})$ & $(0.49-0.56)$ & $32.3 \%$ & $69.4 \%$ & $10.2 \%$ & $90.5 \%$ \\
\hline & (women) & & $(20.9 \%-45.3 \%)$ & $(65.4 \%-73.1 \%)$ & $(6.31 \%-15.2 \%)$ & (87.4\%-93.1\%) \\
\hline \multirow[t]{2}{*}{ Platelets } & $158\left(\times 10^{9} / \mathrm{l}\right)$ & 0.51 & $97.6 \%$ & $3.32 \%$ & $16.3 \%$ & $87.8 \%$ \\
\hline & & $(0.49-0.52)$ & $(94.5 \%-99.2 \%)$ & $(2.34 \%-4.57 \%)$ & $(14.3 \%-18.5 \%)$ & $(73.8 \%-95.9 \%)$ \\
\hline \multicolumn{7}{|c|}{ Population-specific thresholds } \\
\hline Test & Cut point & $\begin{array}{l}\text { AUROC } \\
(95 \% \mathrm{Cl}) \\
\end{array}$ & Sensitivity (\%) $(95 \% \mathrm{Cl})$ & $\begin{array}{c}\text { Specificity (\%) } \\
(95 \% \mathrm{Cl})\end{array}$ & PPV (\%) $(95 \% \mathrm{Cl})$ & NPV (\%) $(95 \% \mathrm{Cl})$ \\
\hline \multirow[t]{2}{*}{ Glucose } & $5.0 \mathrm{mmol} / \mathrm{l}$ & 0.58 & $70.8 \%$ & $45.4 \%$ & $20.0 \%$ & $89.0 \%$ \\
\hline & & $(0.55-0.62)$ & $(64.1 \%-76.9 \%)$ & $(42.4 \%-48.4 \%)$ & $(17.2 \%-23.1 \%)$ & $(86.1 \%-91.5 \%)$ \\
\hline \multirow[t]{4}{*}{ Waist } & 78.8 cms (men) & & $87.7 \%$ & $35.2 \%$ & $28.2 \%$ & $90.8 \%$ \\
\hline & & 0.68 & $(81.0 \%-92.7 \%)$ & $(30.9 \%-39.6 \%)$ & $(24.1 \%-32.5 \%)$ & $(85.8 \%-94.4 \%)$ \\
\hline & $73.5 \mathrm{cms}$ (women & $(0.65-0.71)$ & $72.4 \%$ & $67.9 \%$ & $19.1 \%$ & $95.6 \%$ \\
\hline & & & $(59.1 \%-83.3 \%)$ & $(63.8 \%-71.8 \%)$ & $(14.3 \%-24.8 \%)$ & $(93.2 \%-97.4 \%)$ \\
\hline \multirow[t]{2}{*}{ Triglyceride } & $1.1 \mathrm{mmol} / \mathrm{l}$ & 0.64 & $76.1 \%$ & $52.2 \%$ & $23.5 \%$ & $91.9 \%$ \\
\hline & & $(0.61-0.67)$ & $(69.7 \%-81.7 \%)$ & $(49.2 \%-55.2 \%)$ & $(20.3 \%-26.9 \%)$ & $(89.4 \%-93.9 \%)$ \\
\hline \multirow[t]{4}{*}{ ALT } & $24(\mathrm{IU} / \mathrm{l})$ & & $55.8 \%$ & $60.7 \%$ & $29.2 \%$ & $82.5 \%$ \\
\hline & (men) & 0.54 & (47.4\%-64\%) & $(56.3 \%-65.0 \%)$ & (23.9\%-34.9\%) & (78.3\%-86.2\%) \\
\hline & $13(\mathrm{IU} / \mathrm{I})$ & $(0.51-0.57)$ & $85.5 \%$ & $27.5 \%$ & $11.2 \%$ & $94.6 \%$ \\
\hline & (women) & & (74.2\%-93.1\%) & $(23.9 \%-31.3 \%)$ & $(8.53 \%-14.4 \%)$ & $(90.1 \%-97.5 \%)$ \\
\hline \multirow[t]{2}{*}{ Platelets } & $262\left(\times 10^{9} / \mathrm{l}\right)$ & 0.56 & $47.4 \%$ & $64.9 \%$ & $20.7 \%$ & $86.5 \%$ \\
\hline & & $(0.52-0.60)$ & $(40.4 \%-54.4 \%)$ & $(62.0 \%-67.8 \%)$ & (17.1\%-24.6\%) & (83.9\%-88.8\%) \\
\hline
\end{tabular}

the established criteria are possibly better at identifying people at low risk of, or excluding, fatty liver.

Interestingly, the factors that we have identified as being associated with increased risk of developing fatty liver are also strongly associated with risk of type 2 diabetes either directly, (glucose, triglyceride, ALT and waist circumference) [24], or as a consequence of their association with the metabolic syndrome (platelet count) [25]. This finding clearly emphasizes the importance of shared risk factors for both cardio-metabolic and fatty liver diseases in a general middle-aged population and strongly suggests a common etio-pathogenesis for type 2 diabetes and NAFLD. Although age is a strong risk factor for type 2 diabetes, age was not an independent risk factor for NAFLD in this occupational cohort, perhaps because the age range was fairly narrow and because the effect of age is at least partly mediated through other factors included in our model.

The unexpected finding that platelet count was associated with increased risk of fatty liver may be explained by the fact that platelets are a potential mediator of inflammation [26,27] and some of the individuals with incident fatty liver at follow up may have developed a more severe form of NAFLD with inflammation and 
Table 4 Performance (AUROC and $95 \% \mathrm{Cls}$ ) of combination of baseline tests for identifying incident fatty liver in training data-set and validation data-set

\begin{tabular}{lcr}
\hline & training data-set & validation data-set \\
\hline New thresholds & & 0.71 \\
(A) glucose, waist, triglyceride, ALT, platelets & 0.75 & $(95 \% \mathrm{Cl}: 0.67-0.74)$ \\
& $(95 \% \mathrm{Cl}: 0.72-0.78)$ & \\
Established thresholds & 0.65 & 0.68 \\
(B) glucose, waist, triglyceride, ALT, platelets & $(95 \% \mathrm{Cl}: 0.61-0.69)$ & $(95 \% \mathrm{Cl}: 0.65-0.72)$ \\
\end{tabular}

A) Combining thresholds of glucose, waist, triglyceride, ALT and platelets using new optimized measurement thresholds, and

(B) Combining thresholds of glucose, waist, triglyceride, ALT and platelets using established measurement thresholds.

fibrosis (non alcoholic steatohepatitis). We suggest that research is needed to explore further the relationship between platelet activation and development of NAFLD. Recent findings that both platelet-derived serotonin and platelet release of the key profibrogenic mediators CXC Chemokine Ligand 4 and transforming growth factor- $\beta$ (TGF- $\beta$ ), all support the notion that platelet activation may be involved in NAFLD progression $[27,28]$.

The diagnostic performance of ultrasound, computed tomography (CT), T1-weighted dual-echo magnetic resonance (MR) imaging, and point-resolved proton (hydrogen $1[(1) \mathrm{H}]$ ) MR spectroscopy in the assessment of hepatic steatosis has previously been compared. MR spectroscopic measurements of hepatic fat and ultrasound measurement of liver fat both correlate well with histopathologic steatosis assessment [29]. Furthermore, compared with histopathologic steatosis assessment (as the gold standard), the sensitivity of ultrasound and MR spectroscopy, was $65 \%$ and $91 \%$, respectively, whereas the specificity was $77 \%$ and $87 \%$ respectively. Thus, in establishing an initial diagnosis of NAFLD it is accepted that ultrasound has an acceptable balance of sensitivity and specificity to detect liver fat, particularly where there is $>30 \%$ liver tissue fat infiltration [30]. Although ultrasonography is a reasonably accurate technique for detecting modest amounts of liver fat ( $>30 \%$ liver fat infiltration), ultrasound has poor sensitivity to detect minor amounts of fatty infiltration [30] and it would be important to test the ability of our five simple cardio-metabolic risk factors to predict lesser amounts of liver fat. However, it was not possible for us to undertake this analysis in our prospective study design as an MRS investigation at baseline and at follow up would have been required in 2589 people.

There are several strengths to our study. There is a considerable body of published research that has investigated cross sectional associations of risk factors with liver fat. To our knowledge, no previous studies have described the etiological factors associated with incident NAFLD in a longitudinal study, and then tested the utility of these factors in risk prediction modeling in a general population. Other studies have used more sensitive tests for detecting liver fat, such as the MRS-based study that focused on developing a risk score (for liver fat) [18]; but these investigators employed cross sectional analyses, in a population that was not solely recruited from a general population. Moreover the investigators used measurements of insulin concentrations that are also not readily available to the primary care physician. Recently, using abdominal ultrasonography, Tsuneto et al. [31] examined 1635 Nagasaki atomic bomb survivors (606 men) biennially without fatty liver at baseline, for a mean follow-up of $11.6+/-4.6$ years. In all, 323 (124 men) incident fatty liver cases were diagnosed. The incidence of fatty liver was 19.9/1000 person-years (22.3 for men, 18.6 for women) and peaked in the sixth decade of life. In multivariate analysis, obesity (RR, 2.55; $\mathrm{P}<0.001$ ), hypertriglyceridemia ( $\mathrm{RR}, 1.92 ; \mathrm{P}<0.001)$ and hypertension ( $R R, 1.31 ; \mathrm{P}=0.046$ ) were independently associated with incident fatty liver. Our results are largely in agreement with these findings, and our work on risk prediction extends this work. Tsuneto et al. showed a borderline significant independent association between blood pressure and fatty liver, a finding that was not confirmed in our study. Interestingly, despite the younger age distribution, the incidence rate for fatty liver was higher in our study (at 34.7/1000 person years).

Our study has some limitations. We have used routine clinical data so that although ultrasonography was undertaken by experienced clinical radiologists, a formal assessment of inter-observer variability was not possible. As the incidence rate for NAFLD is probably highest in the sixth decade [31], and because our study population included a relatively narrow age range with a preponderance of men, our estimates of fatty liver incidence cannot be extrapolated to the whole population. We do not have data describing behaviour during the follow up period, and therefore we are unable to comment as to whether there was any change between baseline and follow up measurements that may have affected risk of fatty liver. As ultrasound does not detect liver fat $\leq 30 \%$ fat infiltration, it is possible that some people had small 
amounts of liver fat present at baseline, which was not detectable. We have studied a single population largely comprising one ethnic group and consequently, our results cannot be extrapolated to other ethnic groups. However, our cohort comprises an ethnic group who are typical of a large percentage of the population of Northeast Asia that represents as many as 1.5 billion people. We were unable to exclude subjects taking drugs known to be associated with increased risk of fatty liver as these data were not available, although in this populationbased cohort, this is likely to be a very small percentage of the total cohort.

\section{Conclusions}

We have identified simple baseline etiological factors (waist circumference, glucose, triglyceride and ALT concentrations, and platelet number) that are associated with incident fatty liver over a follow up of an average of 4.4 years. Importantly, we have shown that the diagnostic performance of either the population-specific or the established thresholds used in the definition of the MetS is such that either could be used in clinical practice to focus limited resources and identify a high risk subgroup for fatty liver. We suggest that this selected sub-group should receive a liver ultrasound and may derive particular benefit from lifestyle change and assessment and management of diabetes risk as well as the hepatic disease risk. We suggest that thresholds of these five factors combined, could be used as a screening tool to select people who are particularly likely to, either already have, or to develop fatty liver. Such an approach would improve the use and specificity of ultrasound imaging in a more targeted approach to the diagnosis of fatty liver.

\section{Abbreviations}

NAFLD: nonalcoholic fatty liver disease; MetS: metabolic syndrome; ALT: alanine aminotransaminases; AST: aspartate transaminase; ROC: receiver operator characteristic curves; AUC: area under curve; AUROC: area under receiver operator characteristic curve; PPV: positive predictive value; NPV: negative predictive value; hs-CRP: high-sensitivity C-reactive protein; HDLC: high density lipoprotein cholesterol; SBP: systolic blood pressure; DBP: diastolic blood pressure; TG: triglyceride; BMI: body mass index.

\section{Competing interests}

The authors declare that they have no competing interests.

\section{Author contributions \\ $\mathrm{K}-\mathrm{CS}$ and CDB designed the study and wrote the manuscript. SHW contributed to study design and to writing the manuscript. B-SK, Y-KC and D-I P contributed to the manuscript. SW and SK analysed the data. All authors read and approved the final manuscript.}

\section{Supported by}

This study was partially supported by Samsung Biomedical Research Institute Grant SBRI C-B1-114-1.

\section{Author details}

'Division of Cardiology, Department of Medicine, Kangbuk Samsung Hospital, Sungkyunkwan University School of Medicine, Seoul, Republic of Korea. ${ }^{2}$ Division of Gastroenterology, Department of Medicine, Kangbuk Samsung Hospital, Sungkyunkwan University School of Medicine, Seoul,
Republic of Korea. ${ }^{3}$ Biostatistics Team, Samsung Biomedical Research Institute, Seoul, Republic of Korea. ${ }^{4}$ Centre for Population Health Sciences, University of Edinburgh, IDS Building, Southampton General Hospital, MP 887, Southampton, UK. ${ }^{5}$ Nutrition and Metabolism Unit, Faculty of Medicine, University of Southampton, IDS Building, Southampton General Hospital, MP 887, Southampton, UK. ${ }^{6} \mathrm{NIHR}$ Southampton Biomedical Research Centre, University Hospital Southampton, IDS Building, Southampton General Hospital, MP 887, Southampton, UK. ' Endocrinology and Metabolism Unit, University of Southampton, IDS Building, Southampton General Hospital, MP 887, Tremona Road, Southampton, UKSO166YD. ${ }^{8}$ Division of Cardiology, Kangbuk Samsung, Hospital, Sungkyunkwan University School of Medicine, \#108, Pyung Dong, Jongro-Ku, Seoul 110-746, Republic of Korea.

Received: 24 November 2011 Accepted: 28 June 2012

Published: 6 July 2012

\section{References}

1. Ruhl CE, Everhart JE: Determinants of the association of overweight with elevated serum alanine aminotransferase activity in the United States. Gastroenterology 2003, 124:71-79.

2. Clark JM, Diehl AM: Defining nonalcoholic fatty liver disease: Implications for epidemiologic studies. Gastroenterology 2003, 124:248-250.

3. Schreuder TC, Verwer BJ, van Nieuwkerk CM, Mulder CJ: Nonalcoholic fatty liver disease: an overview of current insights in pathogenesis, diagnosis and treatment. World J Gastroenterol 2008, 14:2474-2486.

4. Neuschwander-Tetri BA, Caldwell SH: Nonalcoholic steatohepatitis: summary of an AASLD Single Topic Conference. Hepatology 2003, 37:1202-1219.

5. Targher G, Bertolini L, Rodella S, Tessari R, Zenari L, Lippi G, Arcaro G: Nonalcoholic fatty liver disease is independently associated with an increased incidence of cardiovascular events in type 2 diabetic patients. Diabetes Care 2007, 30:2119-2121.

6. Marchesini G, Bugianesi E, Forlani G, Cerrelli F, Lenzi M, Manini R, Natale S, Vanni E, Villanova N, Melchionda N, Rizzetto M: Nonalcoholic fatty liver, steatohepatitis, and the metabolic syndrome. Hepatology 2003, 37:917-923.

7. Holt HB, Wild SH, Wood PJ, Zhang J, Darekar AA, Dewbury K, Poole RB, Holt RI, Phillips DI, Byrne CD: Non-esterified fatty acid concentrations are independently associated with hepatic steatosis in obese subjects. Diabetologia 2006, 49:141-148.

8. Vuppalanchi R, Chalasani N: Nonalcoholic fatty liver disease and nonalcoholic steatohepatitis: Selected practical issues in their evaluation and management. Hepatology 2009, 49:306-317.

9. Prati D, Taioli E, Zanella A, Della TE, Butelli S, Del VE, Vianello L, Zanuso F, Mozzi F, Milani S, Conte D, Colombo M, Sirchia G: Updated definitions of healthy ranges for serum alanine aminotransferase levels. Ann Intern Med 2002, 137:1-10.

10. Szczepaniak LS, Babcock EE, Schick F, Dobbins RL, Garg A, Burns DK, McGarry JD, Stein DT: Measurement of intracellular triglyceride stores by H spectroscopy: validation in vivo. Am J Physiol 1999, 276:E977-E989.

11. Cassidy FH, Yokoo T, Aganovic L, Hanna RF, Bydder M, Middleton MS, Hamilton G, Chavez AD, Schwimmer JB, Sirlin CB: Fatty liver disease: MR imaging techniques for the detection and quantification of liver steatosis. Radiographics 2009, 29:231-260.

12. Byrne CD, Olufadi R, Bruce KD, Cagampang FR, Ahmed MH: Metabolic disturbances in non-alcoholic fatty liver disease. Clin Sci (Lond) 2009, 116:539-564.

13. Handberg A, Hojlund K, Gastaldelli A, Flyvbjerg A, Dekker JM, Petrie J, Piatti $P$, Beck-Nielsen H: Plasma sCD36 is associated with markers of atherosclerosis, insulin resistance and fatty liver in a nondiabetic healthy population. J Intern Med 2012, 271:294-304.

14. Gastaldelli A, Kozakova M, Hojlund K, Flyvbjerg A, Favuzzi A, Mitrakou A, Balkau B: Fatty liver is associated with insulin resistance, risk of coronary heart disease, and early atherosclerosis in a large European population. Hepatology 2009, 49:1537-1544.

15. Wang PW, Hsieh CJ, Psang LC, Cheng YF, Liou CW, Weng SW, Chen JF, Chen IY, Li RH, Eng HL: Fatty liver and chronic inflammation in Chinese adults. Diabetes Res Clin Pract 2008, 81:202-208.

16. Bedogni G, Kahn HS, Bellentani S, Tiribelli C: A simple index of lipid overaccumulation is a good marker of liver steatosis. BMC Gastroenterol 2010, 10:98. 
17. Bedogni G, Bellentani S, Miglioli L, Masutti F, Passalacqua M, Castiglione A, Tiribelli C: The Fatty Liver Index: a simple and accurate predictor of hepatic steatosis in the general population. BMC Gastroenterol 2006, 6:33.

18. Kotronen A, Peltonen M, Hakkarainen A, Sevastianova K, Bergholm R, Johansson LM, Lundbom N, Rissanen A, Ridderstrale M, Groop L, OrhoMelander M, Yki-Jarvinen H: Prediction of non-alcoholic fatty liver disease and liver fat using metabolic and genetic factors. Gastroenterology 2009, 137:865-872.

19. Chalasani N: Nonalcoholic fatty liver disease liver fat score and fat equation to predict and quantitate hepatic steatosis: promising but not prime time! Gastroenterology 2009, 137:772-775.

20. Matthews DR, Hosker JP, Rudenski AS, Naylor BA, Treacher DF, Turner RC: Homeostasis model assessment: insulin resistance and beta-cell function from fasting plasma glucose and insulin concentrations in man. Diabetologia 1985, 28:412-419.

21. Saverymuttu SH, Joseph AE, Maxwell JD: Ultrasound scanning in the detection of hepatic fibrosis and steatosis. Br Med J (Clin Res Ed) 1986, 292:13-15.

22. Alberti KG, Eckel RH, Grundy SM, Zimmet PZ, Cleeman Jl, Donato KA, Fruchart JC, James WP, Loria CM, Smith SC Jr: Harmonizing the metabolic syndrome: a joint interim statement of the International Diabetes Federation Task Force on Epidemiology and Prevention; National Heart, Lung, and Blood Institute; American Heart Association; World Heart Federation; International Atherosclerosis Society; and international association for the Study of Obesity. Circulation 2009, 120:1640-1645.

23. Youden WJ: Index for rating diagnostic tests. Cancer 1950, 3:32-35.

24. Wang J, Stancakova A, Kuusisto J, Laakso M: Identification of undiagnosed type 2 diabetic individuals by the finnish diabetes risk score and biochemical and genetic markers: a population-based study of 7232 Finnish men. J Clin Endocrinol Metab 2010, 95:3858-3862

25. Jesri A, Okonofua EC, Egan BM: Platelet and white blood cell counts are elevated in patients with the metabolic syndrome. J Clin Hypertens (Greenwich) 2005, 7:705-711.

26. Ripoche J: Blood platelets and inflammation: Their relationship with liver and digestive diseases. Clin Res Hepatol Gastroenterol 2011, 35:353-357.

27. Semple JW, Italiano JE, Freedman J: Platelets and the immune continuum. Nat Rev Immunol 2011, 11:264-274.

28. Adams LA: Biomarkers of liver fibrosis. J Gastroenterol Hepatol 2011 26:802-809.

29. van Werven JR, Marsman HA, Nederveen AJ, Smits NJ, ten Kate FJ, van Gulik $T M$, Stoker J: Assessment of hepatic steatosis in patients undergoing liver resection: comparison of US, CT, T1-weighted dual-echo MR imaging, and point-resolved 1 H MR spectroscopy. Radiology 2010, 256:159-168,

30. Palmentieri B, de Sio I, La Mura V, Masarone M, Vecchione R, Bruno S, Torella R, Persico M: The role of bright liver echo pattern on ultrasound B-mode examination in the diagnosis of liver steatosis. Dig Liver Dis 2006, 38:485-489.

31. Tsuneto A, Hida A, Sera N, Imaizumi M, Ichimaru S, Nakashima E, Seto S, Maemura K, Akahoshi M: Fatty liver incidence and predictive variables. Hypertens Res 2010, 33:638-643.

doi:10.1186/1471-230X-12-84

Cite this article as: Sung et al: Predicting incident fatty liver using simple cardio-metabolic risk factors at baseline. BMC Gastroenterology 2012 12:84.

\section{Submit your next manuscript to BioMed Central and take full advantage of:}

- Convenient online submission

- Thorough peer review

- No space constraints or color figure charges

- Immediate publication on acceptance

- Inclusion in PubMed, CAS, Scopus and Google Scholar

- Research which is freely available for redistribution 